\section{Rheumatoid arthritis and herpes zoster: risk and prevention in those treated with anti-tumour necrosis factor therapy}

\author{
K L Winthrop, ${ }^{1}$ D E Furst ${ }^{2}$
}

\section{COMMENTARY}

In this issue of Annals of Rheumatic Diseases, Garcia-Doval and colleagues add to a flurry of recent studies examining the risk and outcomes of herpes zoster (HZ) among those patients who use antitumour necrosis factor (TNF) therapies. While the increased risk of infection due to granulomatous and select other intracellular pathogens has been clearly demonstrated in this setting, ${ }^{1-3}$ much less is clear with regard to viral pathogens. ${ }^{4}$ The reactivation of varicella zoster virus ( $\mathrm{HZ}$ or shingles) is of public health concern given HZ's ability to cause substantial long-term pain, depression and disability due to postherpetic neuralgia (PHN). ${ }^{4} \mathrm{HZ}$ is common; approximately one-third of the population will experience it during their lifetime, with $18 \%$ of cases resulting in $\mathrm{PHN}$ and $10 \%$ to $15 \%$ involving ocular tissues with potential for permanent visual loss. Rarely, HZ can disseminate and cause systemic complications and death, a risk heightened by HIV infection and other forms of severe immunosuppression. ${ }^{67} \mathrm{HZ}$ rates increase substantially with age, as cell-mediated immunity to varicella virus wanes and as comorbidities associated with immunosuppression become more common. In the USA, HZ incidence rates range between 4 and 11/1000 patient-years in patients aged 50 and 80 years, respectively, with rates higher in women. ${ }^{67}$

Recently, a live-attenuated vaccine to prevent HZ (Zostavax, Merck, Whitehouse Station, New Jersey, USA) has been developed and approved for use in patients age 60 years or older, regardless

${ }^{1}$ Division of Infectious Diseases, Oregon Health and Science University, Portland, Oregon, USA; ${ }^{2}$ Division of Rheumatology, Geffen School of Medicine at the University of California in Los Angeles, Los Angeles, California, USA

Correspondence to K L Winthrop, 3375 SW Terwilliger Boulevard, Oregon Health and Science University, Portland, OR 97239, USA winthrop@ohsu.edu of their $\mathrm{HZ}$ or varicella history. ${ }^{7}$ For rheumatologists, who frequently treat immunosuppresed individuals within this age group, the questions begin here: are my patients at increased risk for $\mathrm{HZ}$ due to their disease or their therapies? Should I avoid anti-TNF therapies in patients with a history of HZ? Can I vaccinate my patient and when?

\section{THE RISK OF HZ ASSOCIATED WITH RHEUMATOID ARTHRITIS AND ITS THERAPIES}

Garcia-Doval et at examined rates of hospitalised episodes of $\mathrm{HZ}$ and primary varicella infection within the Spanish BIOBADASER database, a registry of patients with rheumatic disease using biological therapy. They documented 106 cases of $\mathrm{HZ}$ among 4655 patients treated with anti-TNF (over 15000 patient-years of follow-up), for an $\mathrm{HZ}$ incidence of 6.5/1000 patient-years in persons using anti-TNF therapy. Of these 106 cases, only 5 required hospitalisation making the rate of hospitalised $\mathrm{HZ}$ $32 / 100000$ patient-years. None of these cases involved disseminated HZ. While rates of hospitalisation in their present study were ninefold higher in patients with rheumatoid arthritis (RA) using anti-TNF therapy as compared to the background Spanish population (they did not compare rates to patients with RA not exposed to biologicals), the authors conclude the absolute risks of hospitalised $\mathrm{HZ}$ are so low that, coupled with the risk of vaccine-related serious adverse events, $\mathrm{HZ}$ vaccination should not be considered prior to anti-TNF therapy. But is this conclusion justifiable?

In the last 2 years, a number of other large population-based studies have evaluated the relative risks of $\mathrm{HZ}$ with anti-TNF and non-biological disease-modifying antirheumatic drug (DMARD) therapy in patients with RA. Smitten et al found the overall rate of $\mathrm{HZ}$ in patients with RA to be more than double that of the general population (9.8 vs 3.7/1000 patient-years), and in a nested case-control study found the HZ risk to be slightly elevated in patients with RA using biological DMARDs (OR 1.54) or traditional DMARDs (OR 1.37) compared to no DMARD therapy. Prednisone use had the highest risk estimate (OR 2.51), and its use in conjunction with DMARDs elevated risk beyond that seen with the DMARDs alone. Strangfeld et a ${ }^{10}$ followed over 5000 patients within the German RABBIT registry and documented $86 \mathrm{HZ}$ episodes for crude incidence rates of 11.1, 8.9 and 5.6 per 1000 patient-years for monoclonal antibody, etanercept and conventional DMARDs, respectively. While a large percentage of these patients were hospitalised (15\%), mostly due to multidermatomal disease, only one had disseminated complications. In multivariate analysis, the rate of $\mathrm{HZ}$ in monoclonal antibody treated individuals was found significantly elevated (HR $1.82(1.05-3.15))$, while the rate of etanercept associated cases was not statistically significantly elevated. In contrast to the Strangfeld et al paper, McDonald and colleagues conducted a large population-based study among 20000 patients with RA within the US Veteran's Affairs health system. While they found a similar HZ incidence of 10/1000 patient-years and also observed an elevated risk with prednisone use, their results were different for those treated with anti-TNF therapy..$^{11}$ Overall, they observed no difference in $\mathrm{HZ}$ risk for those treated with anti-TNF therapy from those treated with non-biological DMARDs. Further, within the anti-TNF treated cohort, they documented statistically significant protective associations for etanercept (HR 0.62 ) and adalimumab (HR 0.53), and a non-significant risk elevation for infliximab (HR 1.32). These investigators also documented a low rate of disseminated complications and hospitalisation for patients treated with anti-TNF who developed HZ during therapy. Lastly, Wolfe et a ${ }^{12}$ did not find an increase HZ risk with anti-TNF therapy (although they did not design their study to necessarily answer this question) and the authors of the present study from BIOBADASAR previously reported elevated (RR 2.7), but not statistically significant, HZ rates of any severity in patients with RA treated with anti-TNF versus non-biological RA comparators. ${ }^{13}$

Collectively, these studies answer some of our questions: yes, HZ risk is 
elevated in RA and it is further elevated 1.5-2.0-fold by prednisone. The inconsistent results for anti-TNF therapy, however, are confusing and in stark contrast to the increased risks observed for antiTNF therapy with tuberculosis and other granulomatous pathogens, where a mechanism of action has been demonstrated in vitro and in animal models. ${ }^{14-16}$ One could hypothesise that TNF antagonistdriven downregulation of interferon $\gamma$ could promote varicella reactivation, however a firm hypothesis and experimental evidence are currently lacking. Further, the low rates of disseminated $\mathrm{HZ}$ observed among anti-TNF users are worth noting. While this could suggest that TNF blockade does not substantially hinder the host's ability to contain HZ once reactivated, it is likely in most cases anti-TNF therapy was stopped and antiviral therapy was employed.

\section{SHOULD I VACCINATE MY PATIENT WITH RA PRIOR TO INITIATION WITH ANTI-TNF THERAPY?}

While we agree that the rates of hospitalised and complicated HZ observed in Garcia-Doval et al's study were low, we disagree with the author's conclusion regarding vaccination and believe the rationale for zoster vaccination goes beyond the goal of simply preventing hospitalised HZ. Clinical trials of Zostavax among largely healthy patients 60 years and older suggest the vaccine decreases $\mathrm{HZ}$ risk by $51 \%$ and $\mathrm{PHN}$ by $66 \%$ in the 3 years post vaccination. ${ }^{717}$ Rates of serious adverse events (SAEs) were similar (1.4\%) between vaccine and placebo groups in the 38000 person phase III Shingles Preventions Study. In a safety substudy, SAEs were more likely to occur in the 42 days post vaccination among vaccinated individuals (RR 1.5, (1.0-2.3)), although these events were infrequent $(1.9 \%$ vaccine vs $1.3 \%$ placebo) and rates of hospitalisation and death were not different between vaccine and placebo groups. ${ }^{7} 17$

Presently, there are no prospective data attesting to the efficacy of $\mathrm{HZ}$ vaccination in patients with RA specifically. However, strong evidence exists arguing for the protective effect and importance of vaccinating those $\geq 60$ years, and given that patients with RA are at higher risk for $\mathrm{HZ}$ and that vaccination with live viruses is contraindicated while using biological therapies, it seems logical to target this group for vaccination before anti-TNF initiation. The intent of vaccination is to lower their risk for 'uncomplicated' $\mathrm{HZ}$ and the significant

- For persons on immunosuppressive therapy, including high-dose corticosteroids ( $\geq 20 \mathrm{mg} /$ day of prednisone or equivalent) lasting 2 or more weeks, Zoster vaccination should be deferred for at least 1 month after discontinuation of such therapy (209).

- Short-term corticosteroid therapy (<14 days); low-to-moderate dose (<20 mg/day of prednisone or equivalent); topical (eg, nasal, skin, inhaled); intra-articular, bursal, or tendon injections; or long-term alternate-day treatment with low to moderate doses of short-acting systemic corticosteroids are not considered to be sufficiently immunosuppressive to cause concerns for vaccine safety. Persons receiving this dose or schedule can receive zoster vaccine.

- herapy with low-doses of methotrexate ( $\leq 0.4 \mathrm{mg} / \mathrm{kg} /$ week), azathioprine $(\leq 3.0 \mathrm{mg} / \mathrm{kg} / \mathrm{day})$, or 6 -mercaptopurine $(\leq 1.5 \mathrm{mg} / \mathrm{kg} /$ day) for treatment of rheumatoid arthritis, psoriasis, polymyositis, sarcoidosis, inflammatory bowel disease and other conditions are also not considered sufficiently immunosuppressive to create vaccine safety concerns and are not contraindications for administration of zoster vaccine.

- Persons receiving recombinant human immune mediators and immune modulators, especially the anti-tumor necrosis factor agents adalimumab, infliximab and etanercept. The safety and efficacy of zoster vaccine administered concurrently with these agents is unknown. If it is not possible to administer zoster vaccine to patients before initiation of therapy, physicians should assess the immune status of the recipient on a caseby-case basis to determine the relevant risks and benefits. Otherwise, vaccination with zoster vaccine should be deferred for at least 1 month after discontinuation of such therapy.

Figure 1 Current Centers for Disease Control (CDC) and Prevention and Advisory Committee on Immunization Practices (ACIP) recommendations for use of the Zostavax for persons using immunosuppressive therapies common in rheumatoid arthritis. ${ }^{7}$ morbidity associated with this common condition, in addition to the rare but more serious manifestations of $\mathrm{HZ}$. Future studies should aim to further elucidate the potential benefits of vaccinating patients with RA specifically, including those younger than 60 years old and those currently receiving various forms of immunosuppressive therapy (figure 1).

Should it be standard of care to consider $\mathrm{HZ}$ vaccination in patients with $\mathrm{RA}$ age $\geq 60$ years prior to anti-TNF initiation or other long-term immunosuppressive regimens? We believe it should.

Funding KLW's work on this manuscript was funded by an Agency for Healthcare Research and Quality (AHRO) grant (1K08HS017552-01). KLW has received a grant from UCB pharmaceuticals and scientific consultant fees from Amgen, Wyeth and Genentech. DEF has received research grants to perform studies with abatacept, adalimumab, certolizumab, etanercept, infliximab, rituximab and tocilizumab, and has consulted with Abbott, Amgen, Bristol Meyer Squibb, Centecor, Genentech, UCB.

Provenance and peer review Commissioned; externally peer reviewed.

Accecpted 25 June 2010

Ann Rheum Dis 2010;69:1735-1737

doi:10.1136/ard.2010.133843

\section{REFERENCES}

1. Winthrop KL. Risk and prevention of tuberculosis and other serious opportunistic infections associated with the inhibition of tumor necrosis factor. Nat Clin Pract Rheumatol 2006:2:602-10.

2. Tubach F, Ravaud P, Salmon-Céron D, et al. Emergence of Legionella pneumophila pneumonia in patients receiving tumor necrosis factor-alpha antagonists. Clin Infect Dis 2006;43:e95-100.

3. Dixon WG, Watson K, Lunt M, et al. Rates of serious infection, including site-specific and bacterial intracellular infection, in rheumatoid arthritis patients receiving anti-tumor necrosis factor therapy: results from the British Society for Rheumatology Biologics Register. Arthritis Rheum 2006;54:2368-76

4. Kim SY, Solomon DH. Tumor necrosis factor blockade and the risk of viral infection. Nat Rev Rheumatol 2010;6:165-74.

5. Schmader KE. Epidemiology and impact on quality of life of postherpetic neuralgia and painful diabetic neuropathy. Clin J Pain 2002;18:350-4.

6. Yawn BP, Saddier P, Wollan PC, et al. A populationbased study of the incidence and complication rates of herpes zoster before zoster vaccine introduction. Mayo Clin Proc 2007:82:1341-9.

7. Harpaz R, Ortega-Sanchez IR, Seward JF.Advisory Committee on Immunization Practices (ACIP) Centers for Disease Control and Prevention (CDC). MMWR Recomm Rep 2008;57(RR-5):1-30; quiz CE2-4.

8. García-Doval I, Pérez-Zafrilla B, Descalzo MA, et al. Incidence and risk of hospitalisation due to shingles and chickenpox in patients with rheumatic diseases treated with TNF antagonists. Ann Rheum Dis 2010;69:1751-55.

9. Smitten AL, Choi HK, Hochberg MC, et al. The risk of herpes zoster in patients with rheumatoid arthritis in the United States and the United Kingdom. Arthritis Rheum 2007:57:1431-8.

10. Strangfeld A, Listing J, Herzer P, et al. Risk of herpes zoster in patients with rheumatoid arthritis 
treated with anti-TNF-alpha agents. JAMA 2009;301:737-44.

11. McDonald JR, Zeringue AL, Caplan L, et al. Herpes zoster risk factors in a national cohort of veterans with rheumatoid arthritis. Clin Infect Dis 2009:48:1364-71.

12. Wolfe F, Michaud K, Chakravarty EF. Rates and predictors of herpes zoster in patients with rheumatoid arthritis and non-inflammatory musculoskeletal disorders. Rheumatology (Oxford) 2006; $45: 1370-5$.
13. Descalzo MA, Biobadaser CC, Biobadaser SG Spanish Registry for adverse events of biologic therapies in rheumatic diseases (BIOBADASER): state report on January 26th 2006. Reumatol Clin 2007:3:4-20.

14. Algood HM, Lin PL, Flynn JL. Tumor necrosis factor and chemokine interactions in the formation and maintenance of granulomas in tuberculosis. Clin Infect Dis 2005;41(Suppl 3):S189-93.

15. Plessner HL, Lin PL, Kohno T, et al. Neutralization of tumor necrosis factor (TNF) by antibody but not TNF receptor fusion molecule exacerbates chronic murine tuberculosis. J Infect Dis 2007;195:1643-50.

16. Marino S, Sud D, Plessner H, et al. Differences in reactivation of tuberculosis induced from anti-TNF treatments are based on bioavailability in granulomatous tissue. PLoS Comput Biol 2007;3:1909-24.

17. Oxman MN, Levin MJ, Johnson GR, et al. A vaccine to prevent herpes zoster and postherpetic neuralgia in older adults. $N \mathrm{Eng} / \mathrm{J}$ Med 2005;352:2271-84. 